\title{
A Novel Spectrum Allocation Strategy with Channel Bonding and Channel Reservation
}

\author{
Shunfu Jin ${ }^{1,2}$, Xinghua Yao ${ }^{1,2}$ and Zhanyou $\mathrm{Ma}^{3}$ \\ ${ }^{1}$ School of Information Science and Engineering \\ Yanshan University, Qinhuangdao 066004 - China \\ ${ }^{2}$ Key Laboratory for Computer Virtual Technology \\ and System Integration of Hebei Province \\ Yanshan University, Qinhuangdao 066004 - China \\ [e-mail: jsf@ysu.edu.cn] \\ ${ }^{3}$ School of Science, Yanshan University \\ Qinhuangdao 066004 - China \\ [e-mail: mzhy55@ysu.edu.cn] \\ *Corresponding author: Shunfu Jin
}

\begin{abstract}
In order to meet various requirements for transmission quality of both primary users (PUs) and secondary users (SUs) in cognitive radio networks, we introduce a channel bonding mechanism for PUs and a channel reservation mechanism for SUs, then we propose a novel spectrum allocation strategy. Taking into account the mistake detection and false alarm due to imperfect channel sensing, we establish a three-dimensional Markov chain to model the stochastic process of the proposed strategy. Using the method of matrix geometric solution, we derive the performance measures in terms of interference rate of PU packets, average delay and throughput of SU packets. Moreover, we investigate the influence of the number of the reserved (resp. licensed) channels on the system performance with numerical experiments. Finally, to optimize the proposed strategy socially, we provide a charging policy for SU packets.
\end{abstract}

Keywords: cognitive radio networks, channel bonding, channel reservation, imperfect channel sensing, three-dimensional Markov chain, charging policy

This work was supported by the Natural Science Foundation of China (61472342). 


\section{Introduction}

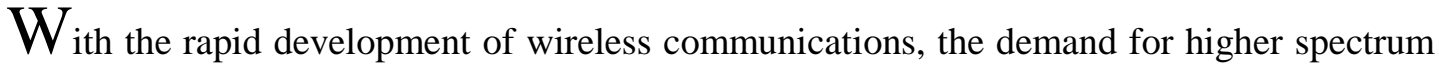
efficiency increases gradually. Cognitive radio (CR) technology has gained more attentions due to its ability to improve the spectrum utilization [1-3]. In cognitive radio networks (CRNs), primary users (PUs) use the licensed spectrum with a higher priority, while secondary users (SUs) can opportunistically access the spectrum hole whenever the spectrum is not occupied during a particular time interval [4-5]. The dynamic spectrum allocation strategy is one of the key techniques to be considered in the design of CRNs [6-9]. Most of the previous researches were carried out under a single channel network environment. In recent years, some scholars hava begun to focus their sights on the spectrum allocation strategies with multiple channels.

Channel bonding strategy, i.e., several available channels are bonded together to transmit one packet, is a popular approach aiming to improve the transmission rate. In [10], Jiao et al. analyzed the channel bonding strategy for SUs. By establishing a two-dimensional continuous-time Markov chain model, they revealed the influence of channel bonding strategy on the forced termination probability and the blocking probability. In [11], Joshi et al. proposed a detailed analytical framework to investigate the system throughput in opportunistic spectrum allocation networks with channel bonding strategy. The analysis results show that the benefits of channel bonding strategy can be realized by adaptively changing the number of the bonded channels. In [12], based on the channel bonding strategy for SUs, Konishi et al. proposed two handoff policies, i.e., the transmission of the SU occupying the most (resp. least) number of the bonded channels will be terminated. Then, they analyzed the performance of these two dynamic spectrum handoff policies. In [13], Balapuwaduge et al. introduced two queues for elastic SU and real-time SU respectively in CRNs with a channel bonding strategy. Furthermore, they evaluated the proposed queueing schemes with continuous-time Markov chain models.

Channel reservation strategy, i.e., a part of the channels are reserved and only can be occupied in a particular case, is also an effective solution to avoid unpredictable interruptions by other users. In [14], Chakraborty et al. proposed a channel reservation strategy for PUs to reduce the probability of their interference with SUs. By developing mathematical models, they obtained the optimal number of channels to be reserved. In [15], Lirio et al. considered channel reservation in the restart retransmission strategy to reduce the interruptions of secondary traffic calls upon the arrival of primary traffic calls, they also quantified the benefits of channel reservation with numerical results. In [16], taking into account several factors in terms of channel reservation, finite capacity of the system buffer and impatience of queued SUs, Wang et al. proposed a framework for admission control and session-level performance analysis. In addition, they constructed a multi-dimensional continuous-time Markov chain to derive the dropping probability and the blocking probability of SUs.

Taking into acount both of the transmission rate of PU packets as well as the throughput of SU packets, we propose a novel spectrum allocation strategy by combining the channel bonding mechanism for PUs with the channel reservation mechanism for SUs. We also established a Markov chain model to evaluate the system performance of CRNs with the proposed strategy. Moreover, we present a charging policy for the spectrum admission of SU packets with imperfect channel sensing.

The rest of this paper is organized as follows. In Section 2, the proposed strategy is described and a three-dimensional Markov chain model is built accordingly. In Section 3, the steady-state distribution is computed. In Section 4, performance measures are derived. Then, 
numerical experiments are presented and discussed. In Section 5, to coordinate the individually optimal behavior and the socially optimal behavior, a charging policy for SU packets is provided. Finally, conclusions are drawn in Section 6.

\section{A Novel Spectrum Allocation Strategy and Markov Chain Model}

In this section, we present a novel spectrum allocation strategy and establish a Markov chain model accordingly.

\subsection{Spectrum Allocation Strategy with Channel Bonding and Channel Reservation}

For application scenery of wireless communications where primary networks and secondary networks coexist, we propose a novel spectrum allocation strategy. In this paper, we consider a spectrum composed of $N$ homo channels. We separate the $N$ channels into two parts: $N_{l}$ licensed channels and $N_{r}$ reserved channels. We have $N=N_{l}+N_{r}, N, N_{l}$ and $N_{r}$ are positive integers. To improve the transmission rate of PU packets, $N_{l}$ licensed channels are bonded together as one for the transmission of a PU packet. To reduce the interruptions by PU packets, $N_{r}$ reserved channels are prepared for the transmissions of SU packets exclusively. Obviously, the more the channels are reserved for SU packets, the less the licensed channels can be bonded for PU packets. Channel reservation will be performed by charging an appropriate admission fee to SU packets. We will carry out the pricing policy in Section 5.3. In addition, we set a buffer big enough to accommodate all the arriving SU packets, while no buffer is prepared for PU packets.

Fig. 1 illustrates the channel allocation mechanism of the novel spectrum allocation strategy.

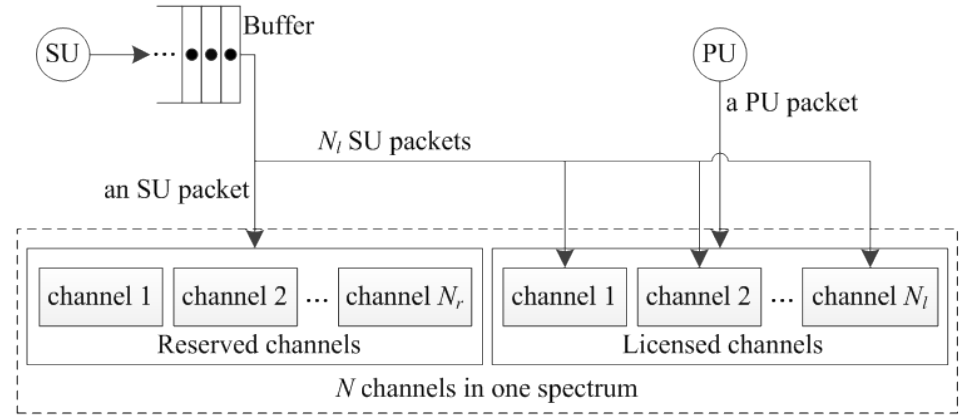

Fig. 1. Channel allocation mechanism

As can be seen in Fig. 1, the PU packets are transmitted only on the licensed channels, and a PU packet occupies all of the $N_{l}$ licensed channels at a time for its transmission, so that a PU packet can be transmitted with a higher transmission rate than on a single channel. The SU packets not only can opportunistically access the licensed channels, but also can complete their transmissions on the reserved channels. On the licensed channels, an SU packet occupies only one channel for its transmission, so at most $N_{l}$ SU packets (if any) can be transmitted simultaneously. On the reserved channels, $N_{r}$ channels are bonded together as one for the transmission of an SU packet. Due to the preemptive priority of PU packets, the transmissions of SU packets on the licensed channels may be interrupted. So, SU packets prefer to access the 
reserved channels rather than the licensed channels if possible. In this way, fewer SU packets will be interrupted by PU packets.

Based on the channel allocation mechanism mentioned above, we discuss the activities of PU packets and SU packets in our proposed strategy with imperfect channel sensing.

1) PU Packets' Activities: When a PU packet arrives at the system, if there is no PU packet on the licensed channels, the newly arriving PU packet will occupy all of the $N_{l}$ licensed channels immediately. Otherwise, the newly arriving PU packet will be blocked by the system. Considering the imperfect channel sensing results, the transmissions of PU packets may be interfered by SU packets. If there occurs a sensing error of mistake detection on the licensed channels, a PU packet will be collided with at most $N_{l}$ SU packets. The interfered PU packet has to leave the system at the disturbance instant.

2) SU Packets' Activities: When an SU packet arrives at the system, it will firstly queue in the buffer waiting for transmission following a first come first serve (FCFS) discipline. Once an SU on the reserved chanels finishes the transmission and the reserved channels are available, the SU packet queueing at the head of the buffer will access the reserved channels immediately. On the other hand, if the reserved channels are occupied, the SU packet queueing in the buffer will try to access the licensed channel. At the beginning instant of each slot, SUs will sense the activities of PU packets and decide whether or not to occupy the licensed channels. The transmissions of the SU packets on the licensed channels are influenced by not only the activities of PU packets but also the sensing results from SUs. If there occurs a false alarm or SUs correctly sense the existence of a PU packet, the SU packets queueing in the buffer will not access the licensed channels, moreover, the SU packets (if any) already on the licensed channels will return back to the head of the buffer. If there occurs a mistake detection on the licensed channels, there will be a collision between a PU packet and at most $N_{l}$ SU packets. The transmissions of the collided SU packets are terminated.

\subsection{Markov Chain Model}

In order to capture the simultanuous events, we consider the discrete-time structure. The time axis is divided into equal intervals called slots. We assume that the arrivals and transmissions for both PU packets and SU packets are based on the slot. The slots are numbered as $n \quad(n=1,2,3 \ldots)$. Let PU packets and SU packets arrive at the system immediately after the beginning instant $n^{+}$of the $n$th slot, the potential arriving interval is marked as $\left(n, n^{+}\right)$. Let PU packets and SU packets depart from the system immediately before the end instant $n^{-}$of the $n$th slot, the potential departure interval is marked as $\left(n^{-}, n\right)$. In other words, an early arrival system is considered.

We define the total number of SU packets in the system as the system level, the condition of the reserved channels as the system phase and the condition of the licensed channels as the system stage. For the instant $n^{+}$, we denote $X_{n}=x \quad(x \geq 0)$ as the system level, $Y_{n}=y$ $(y=0,1)$ as the system phase, and $Z_{n}=z(z=0,1,2,3)$ as the system stage. For the system phase, $y=0$ represents the reserved channels are idle, $y=1$ represents the reserved channels are being occupied by an SU packet. For the system stage, $z=0$ represents all of the licensed channels are idle, $z=1$ represents the licensed channels are occupied by a PU packet, $z=2$ represents at least one of the licensed channels is occupied by SU packets, $z=3$ represents the licensed channels are disordered, i.e., there is a collision on the licensed channels between a PU packet and at least one SU packet. Thus, $\left\{X_{n}, Y_{n}, Z_{n}, n \geq 0\right\}$ constitutes a three-dimensional 
stochastic process.

In addition, we present the following assumptions on the stochastic process.

- The arriving intervals of PU packets and SU packets are supposed to follow Bernoulli processes with parameters $\lambda_{p} \quad\left(0<\lambda_{p}<1, \bar{\lambda}_{p}=1-\lambda_{p}\right) \quad$ and $\lambda_{s}$ $\left(0<\lambda_{s}<1, \bar{\lambda}_{s}=1-\lambda_{s}\right)$, respectively.

- The data rate of each licensed channel is the same as that of each reserved channel. For a single channel, the transmission rates for PU packets and SU packets are supposed to be $\mu_{0}$ and $\mu_{1}$, respectively. Moreover, the transmission time of a PU packet on the $N_{l}$ licensed channels is supposed to follow geometric distribution with parameter $\mu_{p}=N_{l} \mu_{0}\left(0<\mu_{p}<1, \bar{\mu}_{p}=1-\mu_{p}\right)$, and the transmission time of an SU packet on the $N_{r}$ reserved channels is supposed to follow geometric distribution with parameter $\mu_{s}=N_{r} \mu_{1}\left(0<\mu_{p}<1, \bar{\mu}_{s}=1-\mu_{s}\right)$.

- The arriving intervals and transmission time for both two kinds of packets (PU packets and SU packets) are supposed to be independent of each other.

- At the beginning instant of each slot, SUs will sense PUs' activities. For each sensing, a false alarm occurs with probability $p_{f}\left(\bar{p}_{f}=1-p_{f}\right)$ and a mistake detection occurs with probability $p_{m}\left(\bar{p}_{m}=1-p_{m}\right)$.

With these assumptions, the stochastic process $\left\{X_{n}, Y_{n}, Z_{n}, n \geq 0\right\}$ mentioned above is in fact a three-dimensional Markov chain, which can be considered as an analytical model. The big enough buffer for SU packets recalls us the total number $x$ of SU packets in the system can be supposed to be infinite, so the proposed Markov chain model consists of an infinite state space. The state space of this Markov chain is illustrated in Table 1.

Table 1. State space of the three-dimensional Markov chain

\begin{tabular}{|c||c|c|c|c|c|c|c|c|}
\hline & $(x, 0,0)$ & $(x, 0,1)$ & $(x, 0,2)$ & $(x, 0,3)$ & $(x, 1,0)$ & $(x, 1,1)$ & $(x, 1,2)$ & $(x, 1,3)$ \\
\hline \hline$x=0$ & $\sqrt{ }$ & $\sqrt{ }$ & & & & & & \\
\hline$x=1$ & & & $\sqrt{ }$ & $\sqrt{ }$ & $\sqrt{ }$ & $\sqrt{ }$ & & \\
\hline $2 \leq x \leq N_{l}$ & & & $\sqrt{ }$ & $\sqrt{ }$ & $\sqrt{ }$ & $\sqrt{ }$ & $\sqrt{ }$ & $\sqrt{ }$ \\
\hline$x \geq N_{l}+1$ & & & & & $\sqrt{ }$ & $\sqrt{ }$ & $\sqrt{ }$ & $\sqrt{ }$ \\
\hline
\end{tabular}

In Table 1, we mark all the possible states with symbol " $\sqrt{ }$ ” for different cases of system level.

Let $\pi_{i, j, k}$ be the steady-state distribution of the three-dimensional Markov chain. $\pi_{i, j, k}$ can be given as follows:

$$
\pi_{i, j, k}=\lim _{n \rightarrow \infty} P\left\{X_{n}=i, Y_{n}=j, Z_{n}=k\right\}, i=0,1,2 \ldots, j=0,1, k=0,1,2,3
$$

\section{Model Analysis}

We define the system state before the one step transition as the original state, the system state after the one step transition as the destined state. Let $i_{1}$ be the system level of the original state, $i_{2}$ be the system level of the destined state. Considering the activities for the two kinds of packets and the imperfect channel sensing results, we discuss the one step transition probability according to the system states shown in Table $\mathbf{1}$. 
1) The original state $\left(i_{1}, 0,0\right), i_{1}=0$ means that there is no any packet (SU packet or PU packet) in the system. During the one step transition, any departure is impossible. The newly arriving SU packet (if any) will access the reserved channels, while the newly arriving PU packet (if any) will access the licensed channels. As a result, the condition of the reserved channels will be dependent on the arrival of SU packets, while the condition of the licensed channels will be dependent on the arrival of PU packets. The possible transitions from the original state $\left(i_{1}, 0,0\right), i_{1}=0$ to different destined states are given in Table 2.

Table 2. Possible transitions from the original state $\left(i_{1}, 0,0\right)$

\begin{tabular}{|c|c|c|}
\hline Destined state & Transition probability & Cases \\
\hline \hline$\left(i_{2}, 0,0\right)$ & $\bar{\lambda}_{s} \bar{\lambda}_{p}$ & \multirow{2}{*}{$i_{1}=0, i_{2}=0$} \\
\cline { 1 - 2 }$\left(i_{2}, 0,1\right)$ & $\bar{\lambda}_{s} \lambda_{p}$ & \\
\hline$\left(i_{2}, 1,0\right)$ & $\lambda_{s} \bar{\lambda}_{p}$ & \multirow{2}{*}{$i_{1}=0, i_{2}=1$} \\
\hline$\left(i_{2}, 1,1\right)$ & $\lambda_{s} \lambda_{p}$ & \\
\hline
\end{tabular}

2) The original state $\left(i_{1}, 0,1\right), i_{1}=0$ means that a PU packet is transmitted on the licensed channels, but there is no SU packet in the system. For this case, the departure of an SU packet is impossible. The newly arriving SU packet (if any) will access the reserved channels, and the condition of the licensed channels will not be influenced by the newly arriving SU packet. Similar to Item 1), the condition of the reserved channels will be dependent on the arrival of SU packets, while the condition of the licensed channels will be dependent on not only the arrival but also the departure of PU packets. The possible transitions from the original state $\left(i_{1}, 0,1\right), i_{1}=0$ to different destined states are given in Table 3 .

Table 3. Possible transitions from the original state $\left(i_{1}, 0,1\right)$

\begin{tabular}{|c|c|c|}
\hline Destined state & Transition probability & Cases \\
\hline \hline$\left(i_{2}, 0,0\right)$ & $\bar{\lambda}_{s} \bar{\lambda}_{p} \mu_{p}$ & \multirow{2}{*}{$i_{1}=0, i_{2}=0$} \\
\cline { 1 - 2 }$\left(i_{2}, 0,1\right)$ & $\bar{\lambda}_{s}\left(\lambda_{p} \mu_{p}+\bar{\mu}_{p}\right)$ & \\
\hline$\left(i_{2}, 1,0\right)$ & $\lambda_{s} \bar{\lambda}_{p} \mu_{p}$ & \multirow{2}{*}{$i_{1}=0, i_{2}=1$} \\
\hline$\left(i_{2}, 1,1\right)$ & $\lambda_{s}\left(\lambda_{p} \mu_{p}+\bar{\mu}_{p}\right)$ & \\
\hline
\end{tabular}

3) The original state $\left(i_{1}, 0,2\right), 1 \leq i_{1} \leq N_{l}$ means that all of the $i_{1}$ SU packets are transmitted on the licensed channels, but there is no PU packet in the system. We introduce a symbol $Q_{u, v}(u \geq v \geq 0)$ to represent the probability that $u$ SU packets are transmitted on the licensed channels before the one step transition, but $(u-v)$ of these SU packets have been transmitted completely after the one step transition. So, $Q_{u, v}=C_{u}^{v} \mu_{1}^{u-v} \bar{\mu}_{1}^{v}$. Moreover, we denote $U_{i_{1}, i_{2}}$ (resp. $V_{i_{1}, i_{2}}$ ) as the probability that the system level transfers from $i_{1}$ to $i_{2}$ given that no SU packet arrives (resp. an SU packet arrives) during the one step transition. So, $U_{i_{1}, i_{2}}=\bar{\lambda}_{s} Q_{i_{1}, i_{2}}, V_{i_{1}, i_{2}}=\lambda_{s} Q_{i_{1}, i_{2}-1}$. The SU packets trying to access the licensed channels will firstly sense the activities of PU packets, and the system stage of the destined state will be influenced by the sensing results. The possible transitions from the original state $\left(i_{1}, 0,2\right)$, 
$1 \leq i_{1} \leq N_{l}$ to different destined states are given in Table 4 .

4) The original state $\left(i_{1}, 0,3\right), 1 \leq i_{1} \leq N_{l}$ means that the reserved channels are idle, but there is a collision on the licensed channels between $i_{1}$ SU packets and a PU packet. All of the collided packets will leave the system just before the end instant of the current slot, then the system state will be changed to $(0,0,0)$ immediately after the departures. So, the possible transitions from the original state $\left(i_{1}, 0,3\right), 1 \leq i_{1} \leq N_{l}$ are the same as the transitions from the original state $\left(i_{1}, 0,0\right), i_{1}=0$ shown in Table 2 .

Table 4. Possible transitions from the original state $\left(i_{1}, 0,2\right)$

\begin{tabular}{|c|c|c|}
\hline Destined state & Transition probability & Cases \\
\hline$\left(i_{2}, 0,0\right)$ & $U_{i_{1}, 0} \bar{\lambda}_{p}$ & \multirow{2}{*}{$1 \leq i_{1} \leq N_{l}, i_{2}=0$} \\
\hline$\left(i_{2}, 0,1\right)$ & $U_{i_{1}, 0} \lambda_{p}$ & \\
\hline$\left(i_{2}, 1,0\right)$ & $U_{i_{1}, i_{2}} \bar{\lambda}_{p} p_{f}$ & \multirow{2}{*}{$1 \leq i_{2} \leq i_{1} \leq N_{l}$} \\
\hline$\left(i_{2}, 1,1\right)$ & $U_{i_{1}, i_{2}} \lambda_{p} \bar{p}_{m}$ & \\
\hline \multirow{3}{*}{$\left(i_{2}, 1,0\right)$} & $\left(U_{i_{1}, 1} p_{f}+V_{i_{1}, 1}\right) \bar{\lambda}_{p}$ & $1 \leq i_{1} \leq N_{l}, i_{2}=1$ \\
\hline & $\left(U_{i_{1}, i_{2}}+V_{i_{1}, i_{2}}\right) \bar{\lambda}_{p} p_{f}$ & $2 \leq i_{2} \leq i_{1} \leq N_{l}$ \\
\hline & $V_{i_{1}, i_{1}+1} \bar{\lambda}_{p} p_{f}$ & $1 \leq i_{1} \leq N_{l}, i_{2}=i_{1}+1$ \\
\hline \multirow{3}{*}{$\left(i_{2}, 1,1\right)$} & $\left(U_{i_{1}, 1} \bar{p}_{m}+V_{i_{1}, 1}\right) \lambda_{p}$ & $1 \leq i_{1} \leq N_{l}, i_{2}=1$ \\
\hline & $\left(U_{i_{1}, i_{2}}+V_{i_{1}, i_{2}}\right) \lambda_{p} \bar{p}_{m}$ & $2 \leq i_{2} \leq i_{1} \leq N_{l}$ \\
\hline & $V_{i_{1}, i_{1}+1} \lambda_{p} \bar{p}_{m}$ & $1 \leq i_{1} \leq N_{l}, i_{2}=i_{1}+1$ \\
\hline$\left(i_{2}, 1,2\right)$ & $V_{i_{1}, i_{2}} \bar{\lambda}_{p} \bar{p}_{f}$ & \multirow{2}{*}{$1 \leq i_{1} \leq N_{l}, \quad 2 \leq i_{2} \leq N_{l}+1, i_{1} \geq i_{2}-1$} \\
\hline$\left(i_{2}, 1,3\right)$ & $V_{i_{1}, i_{2}} \lambda_{p} p_{m}$ & \\
\hline
\end{tabular}

Table 5. Possible transitions from the original state $\left(i_{1}, 1,0\right)$

\begin{tabular}{|c|c|c|}
\hline Destined state & Transition probability & Cases \\
\hline \hline$\left(i_{2}, 0,0\right)$ & $\bar{\lambda}_{s} \mu_{s} \bar{\lambda}_{p}$ & \multirow{2}{*}{$i_{1}=1, i_{2}=0$} \\
\hline$\left(i_{2}, 0,1\right)$ & $\bar{\lambda}_{s} \mu_{s} \lambda_{p}$ & \\
\hline \multirow{5}{*}{$\left(i_{2}, 1,0\right)$} & $\bar{\lambda}_{s} \mu_{s} \bar{\lambda}_{p}$ & $i_{1}=2, i_{2}=1$ \\
\cline { 2 - 3 } & $\bar{\lambda}_{s} \mu_{s} \bar{\lambda}_{p} p_{f}$ & $i_{1} \geq 3, i_{2}=i_{1}-1$ \\
\cline { 2 - 3 } & $\left(\bar{\lambda}_{s} \bar{\mu}_{s}+\lambda_{s} \mu_{s}\right) \bar{\lambda}_{p}$ & $i_{1}=1, i_{2}=1$ \\
\cline { 2 - 3 } & $\left(\bar{\lambda}_{s} \bar{\mu}_{s}+\lambda_{s} \mu_{s}\right) \bar{\lambda}_{p} p_{f}$ & $i_{1} \geq 2, i_{2}=i_{1}$ \\
\cline { 2 - 3 } & $\lambda_{s} \bar{\mu}_{s} \bar{\lambda}_{p} p_{f}$ & $i_{1} \geq 1, i_{2}=i_{1}+1$ \\
\hline \multirow{5}{*}{$\left(i_{2}, 1,1\right)$} & $\bar{\lambda}_{s} \mu_{s} \lambda_{p}$ & $i_{1}=2, i_{2}=1$ \\
\cline { 2 - 3 } & $\bar{\lambda}_{s} \mu_{s} \lambda_{p} \bar{p}_{m}$ & $i_{1} \geq 3, i_{2}=i_{1}-1$ \\
\cline { 2 - 3 } & $\left(\bar{\lambda}_{s} \bar{\mu}_{s}+\lambda_{s} \mu_{s}\right) \lambda_{p}$ & $i_{1}=1, i_{2}=1$ \\
\cline { 2 - 3 } & $\left(\bar{\lambda}_{s} \bar{\mu}_{s}+\lambda_{s} \mu_{s}\right) \lambda_{p} \bar{p}_{m}$ & $i_{1} \geq 2, i_{2}=i_{1}$ \\
\hline
\end{tabular}




\begin{tabular}{|c|c|c|}
\hline & $\lambda_{s} \bar{\mu}_{s} \lambda_{p} \bar{p}_{m}$ & $i_{1} \geq 1, i_{2}=i_{1}+1$ \\
\hline \multirow{4}{*}{$\left(i_{2}, 1,2\right)$} & $\bar{\lambda}_{s} \mu_{s} \bar{\lambda}_{p} \bar{p}_{f}$ & $i_{1} \geq 3, i_{2}=i_{1}-1$ \\
\cline { 2 - 3 } & $\left(\bar{\lambda}_{s} \bar{\mu}_{s}+\lambda_{s} \mu_{s}\right) \bar{\lambda}_{p} \bar{p}_{f}$ & $i_{1} \geq 2, i_{2}=i_{1}$ \\
\cline { 2 - 3 } & $\lambda_{s} \bar{\mu}_{s} \bar{\lambda}_{p} \bar{p}_{f}$ & $i_{1} \geq 1, i_{2}=i_{1}+1$ \\
\hline \multirow{4}{*}{$\left(i_{2}, 1,3\right)$} & $\bar{\lambda}_{s} \mu_{s} \lambda_{p} p_{m}$ & $i_{1} \geq 3, i_{2}=i_{1}-1$ \\
\cline { 2 - 3 } & $\left(\bar{\lambda}_{s} \bar{\mu}_{s}+\lambda_{s} \mu_{s}\right) \lambda_{p} p_{m}$ & $i_{1} \geq 2, i_{2}=i_{1}$ \\
\cline { 2 - 3 } & $\lambda_{s} \bar{\mu}_{s} \lambda_{p} p_{m}$ & $i_{1} \geq 1, i_{2}=i_{1}+1$ \\
\hline
\end{tabular}

5) The original state $\left(i_{1}, 1,0\right), i_{1} \geq 1$ means that an SU packet is transmitted on the reserved channels, the rest SU packets (if any) queue in the buffer, but the licensed channels are idle. For this case, the departure of a PU packet is impossible, and the system level $i_{2}$ of the destined state will be decreased by one with probability $\bar{\lambda}_{\mathrm{s}} \mu_{\mathrm{s}}$, fixed at $i_{1}$ with probability $\left(\bar{\lambda}_{s} \bar{\mu}_{s}+\lambda_{s} \mu_{s}\right)$, or increased by one with probability $\lambda_{s} \bar{\mu}_{s}$. The possible transitions from the original state $\left(i_{1}, 1,0\right), i_{1} \geq 1$ to different destined states are given in Table 5 .

6) The original state $\left(i_{1}, 1,1\right), i_{1} \geq 1$ means that an SU packet is transmitted on the reserved channels, the rest SU packets (if any) queue in the buffer, and a PU packet is transmitted on the licensed channels. Just as Item 5), for the destined state, the system level can be $i_{2}=i_{1}-1, i_{2}=i_{1}$, or $i_{2}=i_{1}+1$. The possible transitions from the original state $\left(i_{1}, 1,1\right)$, $i_{1} \geq 1$ to different destined states are given in Table 6 .

Table 6. Possible transitions from the original state $\left(i_{1}, 1,1\right)$

\begin{tabular}{|c|c|c|}
\hline Destined state & Transition probability & Cases \\
\hline \hline$\left(i_{2}, 0,0\right)$ & $\bar{\lambda}_{s} \mu_{s} \bar{\lambda}_{p} \mu_{p}$ & \multirow{2}{*}{$i_{1}=1, i_{2}=0$} \\
\hline$\left(i_{2}, 0,1\right)$ & $\bar{\lambda}_{s} \mu_{s}\left(\lambda_{p} \mu_{p}+\bar{\mu}_{p}\right)$ & \\
\hline \multirow{5}{*}{$\left(i_{2}, 1,0\right)$} & $\bar{\lambda}_{s} \mu_{s} \bar{\lambda}_{p} \mu_{p}$ & $i_{1}=2, i_{2}=1$ \\
\cline { 2 - 3 } & $\bar{\lambda}_{s} \mu_{s} \bar{\lambda}_{p} \mu_{p} p_{f}$ & $i_{1} \geq 3, i_{2}=i_{1}-1$ \\
\cline { 2 - 3 } & $\left(\bar{\lambda}_{s} \bar{\mu}_{s}+\lambda_{s} \mu_{s}\right) \bar{\lambda}_{p} \mu_{p}$ & $i_{1}=1, i_{2}=1$ \\
\cline { 2 - 3 } & $\left(\bar{\lambda}_{s} \bar{\mu}_{s}+\lambda_{s} \mu_{s}\right) \bar{\lambda}_{p} \mu_{p} p_{f}$ & $i_{1} \geq 2, i_{2}=i_{1}$ \\
\cline { 2 - 3 } & $\lambda_{s} \bar{\mu}_{s} \bar{\lambda}_{p} \mu_{p} p_{f}$ & $i_{1} \geq 1, i_{2}=i_{1}+1$ \\
\hline \multirow{5}{*}{$\left(i_{2}, 1,1\right)$} & $\bar{\lambda}_{s} \mu_{s}\left(\lambda_{p} \mu_{p}+\bar{\mu}_{p}\right)$ & $i_{1}=2, i_{2}=1$ \\
\cline { 2 - 3 } & $\bar{\lambda}_{s} \mu_{s}\left(\lambda_{p} \mu_{p}+\bar{\mu}_{p}\right) \bar{p}_{m}$ & $i_{1} \geq 3, i_{2}=i_{1}-1$ \\
\cline { 2 - 3 } & $\left(\bar{\lambda}_{s} \bar{\mu}_{s}+\lambda_{s} \mu_{s}\right)\left(\lambda_{p} \mu_{p}+\bar{\mu}_{p}\right)$ & $i_{1}=1, i_{2}=1$ \\
\cline { 2 - 3 } & $\left(\bar{\lambda}_{s} \bar{\mu}_{s}+\lambda_{s} \mu_{s}\right)\left(\lambda_{p} \mu_{p}+\bar{\mu}_{p}\right) \bar{p}_{m}$ & $i_{1} \geq 2, i_{2}=i_{1}$ \\
\cline { 2 - 3 } & $\lambda_{s} \bar{\mu}_{s}\left(\lambda_{p} \mu_{p}+\bar{\mu}_{p}\right) \bar{p}_{m}$ & $i_{1} \geq 1, i_{2}=i_{1}+1$ \\
\hline \multirow{5}{*}{$\left(i_{2}, 1,2\right)$} & $\bar{\lambda}_{s} \mu_{s} \bar{\lambda}_{p} \mu_{p} \bar{p}_{f}$ & $i_{1} \geq 3, i_{2}=i_{1}-1$ \\
\cline { 2 - 3 } & $\left(\bar{\lambda}_{s} \bar{\mu}_{s}+\lambda_{s} \mu_{s}\right) \bar{\lambda}_{p} \mu_{p} \bar{p}_{f}$ & $i_{1} \geq 2, i_{2}=i_{1}$ \\
\cline { 2 - 3 } & $\lambda_{s} \bar{\mu}_{s} \bar{\lambda}_{p} \mu_{p} \bar{p}_{f}$ & $i_{1} \geq 1, i_{2}=i_{1}+1$ \\
\hline
\end{tabular}




\begin{tabular}{|c|c|c|}
\hline \multirow{4}{*}{$\left(i_{2}, 1,3\right)$} & $\bar{\lambda}_{s} \mu_{s}\left(\lambda_{p} \mu_{p}+\bar{\mu}_{p}\right) p_{m}$ & $i_{1} \geq 3, i_{2}=i_{1}-1$ \\
\cline { 2 - 3 } & $\left(\bar{\lambda}_{s} \bar{\mu}_{s}+\lambda_{s} \mu_{s}\right)\left(\lambda_{p} \mu_{p}+\bar{\mu}_{p}\right) p_{m}$ & $i_{1} \geq 2, i_{2}=i_{1}$ \\
\cline { 2 - 3 } & $\lambda_{s} \bar{\mu}_{s}\left(\lambda_{p} \mu_{p}+\bar{\mu}_{p}\right) p_{m}$ & $i_{1} \geq 1, i_{2}=i_{1}+1$ \\
\hline
\end{tabular}

7) The original state $\left(i_{1}, 1,2\right), i_{1} \geq 2$ means that all of the reserved channels and the licensed channels are occupied by SU packets, the rest SU packets (if any) queue in the buffer. For this case, we denote $R_{i_{1}, i_{2}}$ (resp. $T_{i_{1}, i_{2}}$ ) as the probability that the system level transfers from $i_{1}$ to $i_{2}$ after the one step transition given that no SU packet arrives and the SU packet on the reserved channels departs (resp. an SU packet arrives and the SU packet on the reserved channels does not depart). We also denote $S_{i_{1}, i_{2}}$ as the probability that the system level transfers from $i_{1}$ to $i_{2}$ after the one step transition given that no SU packet arrives and the SU packet on the reserved channels does not depart, or an SU packet arrives and the SU packet on the reserved channels departs. So, if $i_{1} \leq N_{l}+1$, then $R_{i_{1}, i_{2}}=\bar{\lambda}_{s} \mu_{s} Q_{i_{1}-1, i_{2}}, T_{i_{1}, i_{2}}=\lambda_{s} \bar{\mu}_{s} Q_{i_{1}-1, i_{2}-2}$, and $S_{i_{1}, i_{2}}=\left(\bar{\lambda}_{s} \bar{\mu}_{s}+\lambda_{s} \mu_{s}\right) Q_{i_{1}-1, i_{2}-1}$; if $i_{1}>N_{l}+1$, then $R_{i_{1}, i_{2}}=R_{N_{l}+1, i_{2}+N_{l}+1-i_{1}}, T_{i_{1}, i_{2}}=T_{N_{l}+1, i_{2}+N_{l}+1-i_{1}}$, and $S_{i_{1}, i_{2}}=S_{N_{l}+1, i_{2}+N_{l}+1-i_{1}}$. The possible transitions from the original state $\left(i_{1}, 1,2\right), i_{1} \geq 2$ to different destined states are given in Table 7.

Table 7. Possible transitions from the original state $\left(i_{1}, 1,2\right)$

\begin{tabular}{|c|c|c|}
\hline Destined state & Transition probability & Cases \\
\hline$\left(i_{2}, 0,0\right)$ & $R_{i, 0} \bar{\lambda}_{p}$ & \multirow{2}{*}{$2 \leq i_{1} \leq N_{l}+1, i_{2}=0$} \\
\hline$\left(i_{2}, 0,1\right)$ & $R_{i, 0} \lambda_{p}$ & \\
\hline$\left(i_{2}, 0,2\right)$ & $R_{i_{1}, i_{2}} \bar{\lambda}_{p} \bar{p}_{f}$ & \multirow{2}{*}{$1 \leq i_{2}<i_{1} \leq N_{l}+1$} \\
\hline$\left(i_{2}, 0,3\right)$ & $R_{i_{1}, i_{2}} \lambda_{p} p_{m}$ & \\
\hline \multirow{10}{*}{$\left(i_{2}, 1,0\right)$} & $\left(R_{i_{1}, 1} p_{f}+S_{i_{1}, 1}\right) \bar{\lambda}_{p}$ & $2 \leq i_{1} \leq N_{l}+1, i_{2}=1$ \\
\hline & $\left(R_{i_{1}, i_{2}}+S_{i_{1}, i_{2}}+T_{i_{1}, i_{2}}\right) \bar{\lambda}_{p} p_{f}$ & $2 \leq i_{2}<i_{1} \leq N_{l}+1$ \\
\hline & $\left(S_{i_{1}, i_{1}}+T_{i_{1}, i_{1}}\right) \bar{\lambda}_{p} p_{f}$ & $2 \leq i_{1} \leq N_{l}+1, i_{2}=i_{1}$ \\
\hline & $T_{i_{1}, i_{1}+1} \bar{\lambda}_{p} p_{f}$ & $2 \leq i_{1} \leq N_{l}+1, i_{2}=i_{1}+1$ \\
\hline & $R_{N_{l}+1,0} \bar{\lambda}_{p}$ & $i_{1}=N_{l}+2, i_{2}=1$ \\
\hline & $R_{N_{l}+1,0} \bar{\lambda}_{p} p_{f}$ & $i_{1} \geq N_{l}+3, i_{2}=i_{1}-N_{l}-1$ \\
\hline & $\left(R_{N_{l}+1,1}+S_{N_{l}+1,1}\right) \bar{\lambda}_{p} p_{f}$ & $i_{1} \geq N_{l}+2, i_{2}=i_{1}-N_{l}$ \\
\hline & $\left(R_{N_{l}+1, m+1}+S_{N_{l}+1, m+1}+T_{N_{l}+1, m+1}\right) \bar{\lambda}_{p} p_{f}$ & $i_{1} \geq N_{l}+2, i_{2}=i_{1}-N_{l}+m, 1 \leq m \leq N_{l}-1$ \\
\hline & $\left(S_{N_{l}+1, N_{l}+1}+T_{N_{l}+1, N_{l}+1}\right) \bar{\lambda}_{p} p_{f}$ & $i_{1} \geq N_{l}+2, i_{2}=i_{1}$ \\
\hline & $T_{N_{l}+1, N_{l}+2} \bar{\lambda}_{p} p_{f}$ & $i_{1} \geq N_{l}+2, i_{2}=i_{1}+1$ \\
\hline \multirow{3}{*}{$\left(i_{2}, 1,1\right)$} & $\left(R_{i_{1}, 1} \bar{p}_{m}+S_{i_{1}, 1}\right) \lambda_{p}$ & $2 \leq i_{1} \leq N_{l}+1, i_{2}=1$ \\
\hline & $\left(R_{i_{1}, i_{2}}+S_{i_{1}, i_{2}}+T_{i_{1}, i_{2}}\right) \lambda_{p} \bar{p}_{m}$ & $2 \leq i_{2}<i_{1} \leq N_{l}+1$ \\
\hline & $\left(S_{i_{1}, i_{1}}+T_{i_{1}, i_{1}}\right) \lambda_{p} \bar{p}_{m}$ & $2 \leq i_{1} \leq N_{l}+1, i_{2}=i_{1}$ \\
\hline
\end{tabular}




\begin{tabular}{|c|c|c|}
\hline & & Refer to Table 7 (continued) \\
\hline Destined state & Transition probability & Cases \\
\hline & $T_{i_{1}, i_{1}+1} \lambda_{p} \bar{p}_{m}$ & $2 \leq i_{1} \leq N_{l}+1, i_{2}=i_{1}+1$ \\
\hline & $R_{N_{l}+1,0} \lambda_{p}$ & $i_{1}=N_{l}+2, i_{2}=1$ \\
\hline & $R_{N_{l}+1,0} \lambda_{p} \bar{p}_{m}$ & $i_{1} \geq N_{l}+3, i_{2}=i_{1}-N_{l}-1$ \\
\hline & $\left(R_{N_{l}+1,1}+S_{N_{l}+1,1}\right) \lambda_{p} \bar{p}_{m}$ & $i_{1} \geq N_{l}+2, i_{2}=i_{1}-N_{l}$ \\
\hline & $\left(R_{N_{l}+1, m+1}+S_{N_{l}+1, m+1}+T_{N_{l}+1, m+1}\right) \lambda_{p} \bar{p}_{m}$ & $i_{1} \geq N_{l}+2, i_{2}=i_{1}-N_{l}+m, 1 \leq m \leq N_{l}-1$ \\
\hline & $\left(S_{N_{l}+1, N_{l}+1}+T_{N_{l}+1, N_{l}+1}\right) \lambda_{p} \bar{p}_{m}$ & $i_{1} \geq N_{l}+2, i_{2}=i_{1}$ \\
\hline & $T_{N_{l}+1, N_{l}+2} \lambda_{p} \bar{p}_{m}$ & $i_{1} \geq N_{l}+2, i_{2}=i_{1}+1$ \\
\hline \multirow{7}{*}{$\left(i_{2}, 1,2\right)$} & $\left(S_{i_{1}, i_{2}}+T_{i_{1}, i_{2}}\right) \bar{\lambda}_{p} \bar{p}_{f}$ & $2 \leq i_{2} \leq i_{1} \leq N_{l}+1$ \\
\hline & $T_{i_{1}, i_{1}+1} \bar{\lambda}_{p} \bar{p}_{f}$ & $2 \leq i_{1} \leq N_{l}+1, i_{2}=i_{1}+1$ \\
\hline & $R_{N_{l}+1,0} \bar{\lambda}_{p} \bar{p}_{f}$ & $i_{1} \geq N_{l}+3, i_{2}=i_{1}-N_{l}-1$ \\
\hline & $\left(R_{N_{l}+1,1}+S_{N_{l}+1,1}\right) \bar{\lambda}_{p} \bar{p}_{f}$ & $i_{1} \geq N_{l}+2, i_{2}=i_{1}-N_{l}$ \\
\hline & $\left(R_{N_{l}+1, m+1}+S_{N_{l}+1, m+1}+T_{N_{l}+1, m+1}\right) \bar{\lambda}_{p} \bar{p}_{f}$ & $i_{1} \geq N_{l}+2, i_{2}=i_{1}-N_{l}+m, 1 \leq m \leq N_{l}-1$ \\
\hline & $\left(S_{N_{l}+1, N_{l}+1}+T_{N_{l}+1, N_{l}+1}\right) \bar{\lambda}_{p} \bar{p}_{f}$ & $i_{1} \geq N_{l}+2, i_{2}=i_{1}$ \\
\hline & $T_{N_{l}+1, N_{l}+2} \bar{\lambda}_{p} \bar{p}_{f}$ & $i_{1} \geq N_{l}+2, i_{2}=i_{1}+1$ \\
\hline \multirow{7}{*}{$\left(i_{2}, 1,3\right)$} & $\left(S_{i_{1}, i_{2}}+T_{i_{1}, i_{2}}\right) \lambda_{p} p_{m}$ & $2 \leq i_{2} \leq i_{1} \leq N_{l}+1$ \\
\hline & $T_{i_{1}, i_{1}+1} \lambda_{p} p_{m}$ & $2 \leq i_{1} \leq N_{l}+1, i_{2}=i_{1}+1$ \\
\hline & $R_{N_{l}+1,0} \lambda_{p} p_{m}$ & $i_{1} \geq N_{l}+3, i_{2}=i_{1}-N_{l}-1$ \\
\hline & $\left(R_{N_{l}+1,1}+S_{N_{l}+1,1}\right) \lambda_{p} p_{m}$ & $i_{1} \geq N_{l}+2, i_{2}=i_{1}-N_{l}$ \\
\hline & $\left(R_{N_{l}+1, m+1}+S_{N_{l}+1, m+1}+T_{N_{l}+1, m+1}\right) \lambda_{p} p_{m}$ & $i_{1} \geq N_{l}+2, i_{2}=i_{1}-N_{l}+m, 1_{1} \leq m \leq N_{l}-1$ \\
\hline & $\left(S_{N_{l}+1, N_{l}+1}+T_{N_{l}+1, N_{l}+1}\right) \lambda_{p} p_{m}$ & $i_{1} \geq N_{l}+2, i_{2}=i_{1}$ \\
\hline & $T_{N_{l}+1, N_{l}+2} \lambda_{p} p_{m}$ & $i_{1} \geq N_{l}+2, i_{2}=i_{1}+1$ \\
\hline
\end{tabular}

8) The original state $\left(i_{1}, 1,3\right), i_{1} \geq 2$ means that the reserved channels are occupied by an SU packet and the licensed channels are disordered, the rest SU packets (if any) queue in the buffer. All of the collided packets will leave the system just before the end instant of the current slot. If $2 \leq i_{1} \leq N_{l}+1$, the system state will be changed to $(1,1,0)$ immediately after the departures, so the possible transitions from the original state $\left(i_{1}, 1,3\right), 2 \leq i_{1} \leq N_{l}+1$ are the same as the transitions from the original state $\left(i_{1}, 1,0\right), i_{1}=1$ shown in Table 5. If $i_{1} \geq N_{l}+2$, the system state will be changed to $\left(i_{1}-N_{l}, 1,0\right)$ immediately after the departures, so the possible transitions from the original state $\left(i_{1}, 1,3\right), i_{1} \geq N_{l}+2$ are the same as the transitions from the original state $\left(i_{1}-N_{l}, 1,0\right), i_{1} \geq N_{l}+2$ shown in Table 5.

Let $\boldsymbol{P}$ be the one step state transition probability matrix of the stochastic process $\left\{X_{n}, Y_{n}, Z_{n}\right\}, \boldsymbol{P}\left(i_{1}, i_{2}\right)$ be the one step transition probability sub-matrix from the system level $i_{1}$ to $i_{2}$. Up to now, all the possible transition probabilities of the Markov chain have been 
given in Tables. 2-7, and the probabilities for the impossible transitions are treated as 0 in matrix $\boldsymbol{P}$. From Tables. 5-7, we conclude that if $i_{1} \geq N_{l}+3$, then $\boldsymbol{P}\left(i_{1}+h, i_{2}+h\right)=\boldsymbol{P}\left(i_{1}, i_{2}\right)$, $h \geq 1$. In other words, from the system level $i_{1}\left(i_{1} \geq N_{l}+3\right)$, all the sub-matrixes $\boldsymbol{P}\left(i_{1}, i_{2}\right)$ begin to be repeated. Introducing matrix notation $\boldsymbol{A}_{i}\left(0 \leq i \leq N_{l}+2\right)$ and letting $\boldsymbol{A}_{i}=\boldsymbol{P}\left(N_{l}+3, N_{l}+4-i\right)$, we structure a matrix equation $\boldsymbol{G}=\sum_{j=0}^{N_{l}+2} \boldsymbol{G}^{j} \boldsymbol{A}_{j}$. When there is a minimal nonnegative solution $\boldsymbol{G}$ and the spectral radius is less than 1 , we can get the steady-state distribution $\pi_{i, j, k}$ defined in Eq. (1) with numerical results by employing the matrix-geometric solution method [17].

\section{Performance Measures and Numerical Experiments}

In this section, we derive performance measures and provide numerical experiments to evaluate the novel spectrum allocation strategy proposed in this paper.

\subsection{Performance Measures}

The interference rate of PU packets is defined as the number of PU packets interfered by SU packets per slot. When a mistake detection occurs, the PU packet in the system will be collided with SU packets, and the licensed channels will be disordered, i.e., the system stage will be $k=3$. Therefore, the interference rate $\theta$ of PU packets is given as follows:

$$
\theta=\sum_{i=1}^{N_{l}} \pi_{i, 0,3}+\sum_{i=2}^{\xi} \pi_{i, 1,3}
$$

where $\xi$ is an integer big enough to satisfy $1-\sum_{i=0}^{\xi} \sum_{j=0}^{1} \sum_{k=0}^{3} \pi_{i, j, k}<\varepsilon$ for achieving an ideal accuracy, and $\varepsilon$ is an arbitrary small value related to the estimation.

The delay of an SU packet is defined as the time period from the instant that an SU packet arrives at the system to the instant that the SU packet departs from the system. According to Little's formula [18], the average delay $\omega$ of SU packets is given as follows:

$$
\omega=\frac{1}{\lambda_{s}}\left(\sum_{i=1}^{N_{l}} i\left(\pi_{i, 0,2}+\pi_{i, 0,3}\right)+\sum_{i=1}^{\xi} i\left(\pi_{i, 1,0}+\pi_{i, 1,1}\right)+\sum_{i=2}^{\xi} i\left(\pi_{i, 1,2}+\pi_{i, 1,3}\right)\right) .
$$

The throughput of SU packets is defined as the number of SU packets transmitted successfully per slot. An SU packet, once joins the buffer, will depart from the system with two cases: One is to be transmitted successfully; The other is to be collided with a PU packet. The system state $(i, 0,3), 1 \leq i \leq N_{l}$ means $i$ SU packets are collided with a PU packet, the system state $(i, 1,3), 2 \leq i \leq N_{l}+1$ means $(i-1)$ SU packets are collided with a PU packet, and the system state $(i, 1,3), i \geq N_{l}+2$ means $N_{l}$ SU packets are collided with a PU packet. Therefore, the throughput $\phi$ of SU packets is given as follows:

$$
\phi=\lambda_{s}-\left(\sum_{i=1}^{N_{l}} i \pi_{i, 0,3}+\sum_{i=2}^{N_{l}+1}(i-1) \pi_{i, 1,3}+\sum_{i=N_{l}+2}^{\xi} N_{l} \pi_{i, 1,3}\right) .
$$




\subsection{Numerical Experiments}

In our proposed spectrum allocation strategy, the total number of the reserved channels and the licensed channels is fixed. When the number of the reserved channels increases (resp. decreases), the number of the licensed channels will decrease (resp. increase). From the view point of the reserved channels, we provide numerical experiments with analysis and simulation to investigate the system performance.

The experiment is carried out with Visual $\mathrm{C}++6.0$ and MATLAB 7.0. The processor is Inter(R) Pentium(R) CPU G620, the operation frequency is $2.60 \mathrm{GHz}$, and the memory is 4 GB. Moreover, the common parameters used in the numerical experiments are summarized in Table 8.

When a false alarm occurs, there is no PU packet on the licensed channels at all, so the false alarm probability $p_{f}$ will not influence directly the interference rate $\theta$ of PU packets. Fig. 2 shows how the interference rate $\theta$ of PU packets changes with respect to the number $N_{r}$ of the reserved channels for different arrival rate $\lambda_{p}$ of PU packets, arrival rate $\lambda_{s}$ of SU packets and mistake detection probability $p_{m}$.

Table 8. Common parameters in the numerical experiments

\begin{tabular}{|c|c|}
\hline Parameters & Values \\
\hline slot & $1 \mathrm{~ms}$ \\
\hline transmission rate on one channel & $11 \mathrm{Mbps}$ \\
\hline total number of the channels in a spectrum & 8 \\
\hline arrival rate of SU packets & $0.1-0.7$ \\
\hline mean size of an SU packet & 2160 Byte \\
\hline arrival rate of PU packets & $0.1-0.2$ \\
\hline mean size of a PU packet & 1440 Byte \\
\hline mistake detection probability & $0.04-0.1$ \\
\hline false alarm probability & $0.01-0.1$ \\
\hline simulation scale & 500,000 slots \\
\hline $\begin{array}{l}\lambda_{p}=0.12, \lambda_{s}=0.65, p_{m}=0.05 \\
\lambda_{p}=0.1, \lambda_{s}=0.65, p_{m}=0.05\end{array}$ & * \\
\hline
\end{tabular}

Fig. 2. Interference rate $\theta$ of PU packets vs. number $N_{r}$ of the reserved channels

From Fig. 2, we observe that for the same arrival rate $\lambda_{p}$ of PU packets, the same arrival rate $\lambda_{s}$ of SU packets and the same mistake detection probability $p_{m}$, the interference rate $\theta$ of PU packets will firstly increase and then decrease as the number $N_{r}$ of the reserved channels increases. When the number of the reserved channels is smaller, most of the SU 
packets will be transmitted on the licensed channels. As the number of the reserved channels increases, the number of the licensed channels will be smaller. Then the transmission rate of a PU packet on the licensed channels will decrease, and the transmission time of a PU packet will be longer accordingly, which will improve the possibility that a PU packet is collided with SU packets. When the number of the reserved channels increases continuously, most of the SU packets will be transmitted on the reserved channels, fewer SU packets will interfere the transmissions of PU packets, so the interference rate of PU packets will decrease.

We also notice that for the same number $N_{r}$ of the reserved channels, when the arrival rate $\lambda_{s}$ (resp. $\lambda_{p}$ ) of $\mathrm{SU}$ (resp. PU) packets and the mistake detection probability $p_{m}$ are given, the interference rate $\theta$ of PU packets will increase as the arrival rate $\lambda_{p}$ (resp. $\lambda_{s}$ ) of $\mathrm{PU}$ (resp. SU) packets increases. The reason is that the bigger the arrival rate of PU (resp. SU) packets is, the more PU (resp. SU) packets will access the system within a certain time interval, then the possibility that the transmission of a PU packet is interfered will be greater.

Furthermore, we find that for the same number $N_{r}$ of the reserved channels, when the arrival rate $\lambda_{p}$ of PU packets and the arrival rate $\lambda_{s}$ of SU packets are given, the interference rate $\theta$ of PU packets will increase along with the mistake detection probability $p_{m}$. This is because that the greater the mistake detection probability is, the more likely is that a PU packet will be collided with SU packets, so the interference rate of PU packets will increase.

The false alarm only makes some SU packets lose their opportunities to be transmitted on the licensed channels, but these SU packets will not be damaged. Thus, the false alarm probability $p_{f}$ will not influence directly the throughput $\phi$ of SU packets. Fig. 3 shows how the throughput $\phi$ of SU packets changes along with the number $N_{r}$ of the reserved channels for different arrival rate $\lambda_{p}$ of PU packets, arrival rate $\lambda_{s}$ of SU packets and mistake detection probability $p_{m}$.

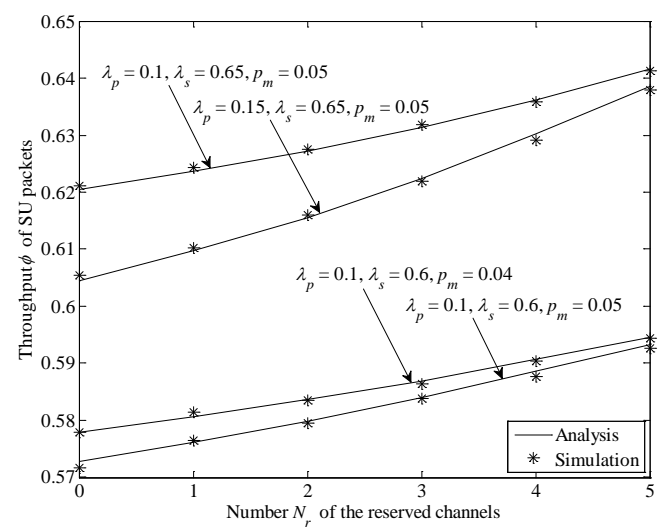

Fig. 3. Throughput $\phi$ of SU packets vs. number $N_{r}$ of the reserved channels

From Fig. 3, we observe that for the same arrival rate $\lambda_{p}$ of PU packets, the same arrival rate $\lambda_{s}$ of SU packets and the same mistake detection probability $p_{m}$, the throughput $\phi$ of SU packets will increase as the number $N_{r}$ of the reserved channels increases. When no channel is reserved for SU packets, SU packets can only opportunistically access the licensed channels. On the other hand, more channels are reserved for SU packets means more SU packets can be transmitted on the reserved channels. Since the transmissions of SU packets on 
the reserved channels will not be interrupted by PU packets, all the SU packets occupying the reserved channels can be transmitted successfully. Therefore, the throughput of SU packets will increase accordingly.

We also notice that for the same number $N_{r}$ of the reserved channels, when the arrival rate $\lambda_{s}$ of SU packets and the mistake detection probability $p_{m}$ are given, the throughput $\phi$ of SU packets will decrease as the arrival rate $\lambda_{p}$ of PU packets increases. The larger the arrival rate of PU packets is, the more likely is that the transmissions of SU packets on the licensed channels will be interrupted by PU packets, then less SU packets will be transmitted successfully, i.e., the throughput of SU packets will decrease.

Moreover, we see that for the same number $N_{r}$ of the reserved channels, when the arrival rate $\lambda_{p}$ of PU packets and the mistake detection probability $p_{m}$ are given, the throughput $\phi$ of SU packets will increase along with the arrival rate $\lambda_{s}$ of SU packets. The reason is that the bigger the arrival rate of SU packets is, the more SU packets will join the system and will be transmitted successfully, so the greater the throughput of SU packets will be.

Furthermore, we find that for the same number $N_{r}$ of the reserved channels, when the arrival rate $\lambda_{p}$ of PU packets and the arrival rate $\lambda_{s}$ of SU packets are given, as the mistake detection probability $p_{m}$ increases, the throughput $\phi$ of SU packets will decrease. This is because that the greater the mistake detection probability is, the more likely is that an SU packet will be collided with a PU packet, then the throughput of SU packets will decrease.

Fig. 4 shows how the average delay $\omega$ of SU packets changes with respect to the number $N_{r}$ of the reserved channels for different arrival rate $\lambda_{p}$ of PU packets, arrival rate $\lambda_{s}$ of SU packets, mistake detection probability $p_{m}$ and false alarm probability $p_{f}$.

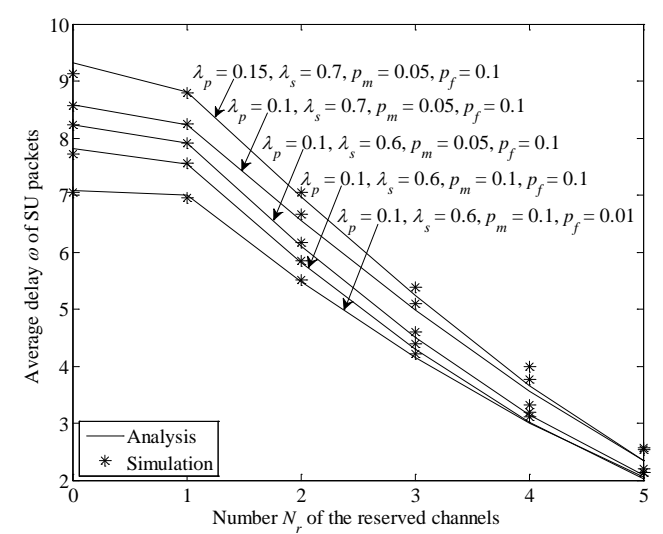

Fig. 4. Average delay $\omega$ of SU packets vs. number $N_{r}$ of the reserved channels

From Fig. 4, we observe that for the same arrival rate $\lambda_{p}$ of PU packets, the same arrival rate $\lambda_{s}$ of SU packets, the same mistake detection probability $p_{m}$ and the same false alarm probability $p_{f}$, the average delay $\omega$ of SU packets will decrease as the number $N_{r}$ of the reserved channels increases. When no channel is reserved for SU packets, SU packets can only opportunistically access the licensed channels. Since PU packets have higher priority than SU packets to occupy the licensed channels, the transmissions of SU packets on the licensed channels are possible to be interrupted due to the arrival of a PU packet. The interrupted SU packets will return back to the buffer for their retransmissions. As the number of the channels 
reserved for SU packets increases, more SU packets can be transmitted on the reserved channels without interruption. Therefore, the average delay of SU packets will decrease.

We also notice that for the same number $N_{r}$ of the reserved channels, when the arrival rate $\lambda_{s}$ of SU packets, the mistake detection probability $p_{m}$ and the false alarm probability $p_{f}$ are given, the average delay $\omega$ of SU packets will increase along with the arrival rate $\lambda_{p}$ of PU packets. The increasing arrival rate of PU packets means that more PU packets will access the licensed channels, then SU packets will have fewer opportunities to occupy the licensed channels. So SU packets will sojourn in the system for a longer time, i.e., the average delay of SU packets will increase.

Moreover, we see that for the same number $N_{r}$ of the reserved channels, when the arrival rate $\lambda_{p}$ of PU packets, the mistake detection probability $p_{m}$ and the false alarm probability $p_{f}$ are given, the average delay $\omega$ of SU packets will increase as the arrival rate $\lambda_{s}$ of SU packets increases. The increasing arrival rate of SU packets means that more SU packets will queue in the buffer, i.e., SU packets will wait in the buffer for a longer time. So the average delay of SU packets will increase.

Furthermore, we find that for the same number $N_{r}$ of the reserved channels, when the arrival rate $\lambda_{p}$ of PU packets, the arrival rate $\lambda_{s}$ of SU packets and the false alarm probability $p_{f}$ are given, as the mistake detection probability $p_{m}$ increases, the average delay $\omega$ of SU packets will decrease. The mistake detection will lead a collision between at most $N_{l}$ SU packets and a PU packet, all of the collided packets will leave the system just before the end instant of the current slot. As the mistake detection probability increases, both the transmission time of these collided SU packets and the waiting time of other SU packets will be shorter. Therefore, the average delay of SU packets will be smaller.

In addition, we detect that for the same number $N_{r}$ of the reserved channels, when the arrival rate $\lambda_{p}$ of PU packets, the arrival rate $\lambda_{s}$ of SU packets and the mistake detection probability $p_{m}$ are given, the average delay $\omega$ of SU packets will increase along with the false alarm probability $p_{f}$. The false alarm will make some SU packets queue in the buffer even the licensed channels are idle. So the bigger the false alarm probability is, the more likely is that the SU packets will wait in the buffer, then the greater the average delay of SU packets will be.

From the numerical experiments shown in Figs. 2-4, we see that the analysis results match well with the simulation results. We also observe that the increasing (resp. decreasing) number of the reserved (resp. licensed) channels in our proposed strategy will lead to results that the interference rate of PU packets firstly increases and then decreases, the throughput of SU packets increases and the average delay of SU packets decreases. The observation means that there is a trade-off between different performance measures. Compared with conventional spectrum allocation strategy considering pure channel bonding or channel reservation, both the requirements of PU packets and SU packets are taken into account in our proposed strategy. Additionally, we conclude that the imperfect channel sensing results, especially mistake detection, influence the system performance greatly, so the sensing errors can not be ignored.

\section{Performance Optimization and Charging Policy for SU packets}

In this section, we firstly give some assumptions for performance optimization. Then we respectively discuss the individually optimal behavior for each SU packet with a best response 
against itself, and the socially optimal behavior for the novel spectrum allocation strategy with the greatest welfare. At last, we provide a charging policy for SU packets by coordinating the two optimal behaviors.

\subsection{Assumptions for Performance Optimization}

For the proposed novel spectrum allocation strategy, an SU packet departs from the system with two cases: The SU packet is transmitted successfully either on the reserved channels or on the licensed channels; The SU packet is collided with a PU packet on the licensed channels, then the collided packets will leave the system at that slot. If a PU packet is collided with SU packets, the transmission of this PU packet is interfered. Based on the Markov chain model established in Subsection 2.2, we add some more assumptions as follows:

- A newly arriving SU packet is not aware of the queue length in the buffer.

- $\quad$ The reward for successful transmission of an SU packet is $R$.

- The cost of an SU packet staying in the system is $C_{s}$ per slot, no matter whether or not the SU packet is transmitted successfully.

- The cost of a PU packet for its transmission to be interfered is $C_{p}$.

\subsection{Individually and Socially Optimal Behaviors}

Firstly, we discuss the individually optimal behavior for each SU packet. With the assumptions given in Subsection 5.1, the individual net benefit $W_{i}$ of an SU packet arrived at the system is given as follows:

$$
W_{i}=\frac{\phi}{\lambda_{s}} R-\omega C_{s}
$$

where $\phi$ is the throughput of SU packets, and $\omega$ is the average delay of SU packets.

In CRNs, each SU packet attempts to join the system to gain benefit [19]. From Fig. 4, we find that as the arrival rate of SU packets increases, the average delay of SU packets increases accordingly. So, the increasing arrival rate of SU packets will lead to a decrease in the individual net benefit of an SU packet. When the individual net benefit $W_{i}$ of an SU packet is zero, i.e., $W_{i}=0$, the system will reach a Nash equilibrium state. The arrival rate of SU packets at the Nash equilibrium state is the individually optimal arrival rate $\lambda_{s}^{e}$.

Then, we discuss the socially optimal behavior for the novel spectrum allocation strategy proposed in this paper. Due to the imperfect channel sensing, SU packets trying to access the licensed channels are possible to interfere the transmissions of PU packets. We define the social welfare as the total net benefits of SU packets arrived at the system minus the total cost of PU packets because of the transmission interference. Therefore, the social welfare $W_{s}$ is given as follows:

$$
W_{s}=\lambda_{s}\left(\frac{\phi}{\lambda_{s}} R-\omega C_{s}\right)-\theta C_{p}
$$

where $\theta$ is the interference rate of PU packets, and $C_{p}$ is the cost of a PU packet for the transmission interference.

The socially optimal arrival rate $\lambda_{s}^{*}$ with the maximum social welfare $W_{s}$ can be denoted as follows: 


$$
\lambda_{s}^{*}=\arg \max _{0<\lambda_{s}<1}\left\{\lambda_{s}\left(\frac{\phi}{\lambda_{s}} R-\omega C_{s}\right)-\theta C_{p}\right\}
$$

Using the parameters given in Table 8, and setting $N_{r}=2, R=18, C_{s}=2.9, C_{p}=5$ as an example, we provide numerical experiments to explore the individual net benefit $W_{i}$ and the social welfare $W_{s}$, respectively.

Fig. 5 demonstrates how the individual net benefit $W_{i}$ of an SU packet changes along with the arrival rate $\lambda_{s}$ of SU packets for different arrival rate $\lambda_{p}$ of PU packets, false alarm probability $p_{f}$ and mistake detection probability $p_{m}$.

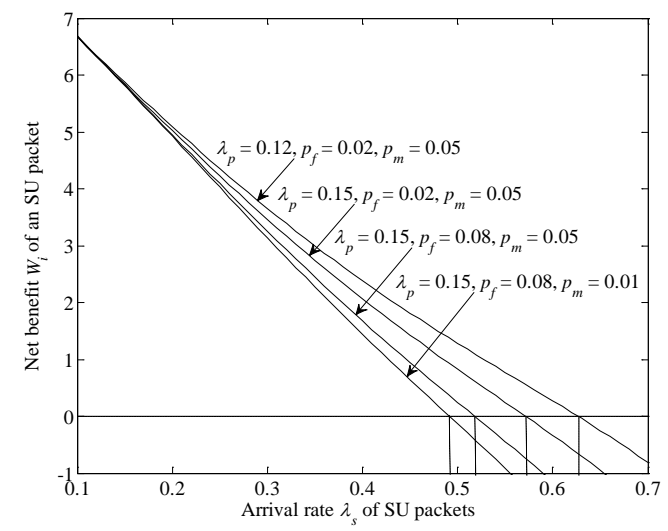

Fig. 5. Individual net benefit $W_{i}$ of an SU packet vs. arrival rate $\lambda_{s}$ of SU packets

As shown in Fig. 5, for all the combinations of arrival rate $\lambda_{p}$ of PU packets, false alarm probability $p_{f}$ and mistake detection probability $p_{m}$, the individual net benefit $W_{i}$ of an SU packet presents a monotone decreasing trend as the arrival rate $\lambda_{s}$ of SU packets increases. We can also see that for all the cases, there is always an individually optimal arrival rate $\lambda_{s}^{e}$ with $W_{i}=0$.

Fig. 6 reveals how the social welfare $W_{s}$ changes with respect to the arrival rate $\lambda_{s}$ of SU packets for different arrival rate $\lambda_{p}$ of PU packets, false alarm probability $p_{f}$ and mistake detection probability $p_{m}$.

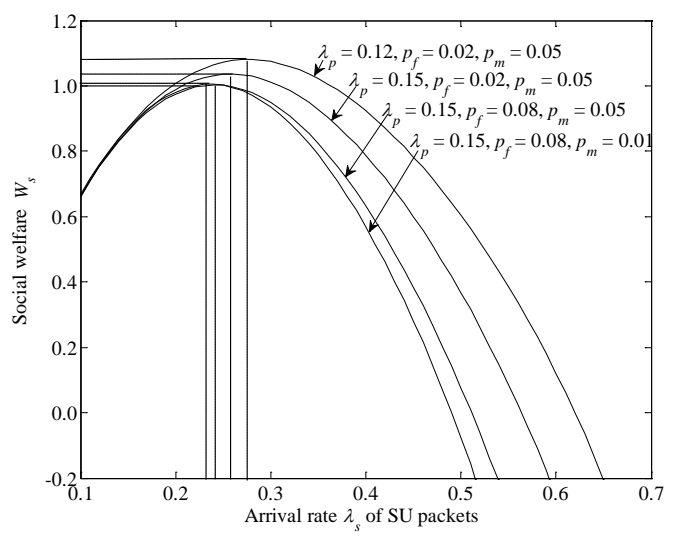

Fig. 6. Social welfare $W_{s}$ vs. arrival rate $\lambda_{s}$ of SU packets 
As shown in Fig. 6, for all the combinations of arrival rate $\lambda_{p}$ of PU packets, false alarm probability $p_{f}$ and mistake detection probability $p_{m}$, the social welfare $W_{s}$ firstly increases and then decreases as the arrival rate $\lambda_{s}$ of SU packets increases. Therefore, there is always a socially optimal arrival rate $\lambda^{*}$ with the maximum social welfare $W_{s}$ for all the cases.

Comparing the results shown in Figs. 5 and 6, we observe that for the same arrival rate $\lambda_{p}$ of PU packets, the same false alarm probability $p_{f}$ and the same mistake detection probability $p_{m}$, the individually optimal arrival rate $\lambda_{s}^{e}$ is always bigger than the socially optimal arrival rate $\lambda_{s}^{*}$, i.e., $\lambda_{s}^{e}>\lambda_{s}^{*}$. That is to say, there will be more SU packets arriving at the system under the individually optimal behavior than under the socially optimal behavior.

\subsection{Charging Policy for SU Packets}

In order to oblige the individually optimal behavior to comply with the socially optimal behavior, we provide a charging policy for SU packets, i.e, charging an appropriate admission fee $F$ to SU packets. The new individual net benefit $W_{i}^{*}$ of an SU packet with the admission fee $F$ can be given as follows:

$$
W_{i}^{*}=\frac{\phi}{\lambda_{s}} R-\omega C_{s}-F
$$

To reach the Nash equilibrium state under the socially optimal behavior, we set $W_{i}^{*}=0$ and $\lambda_{s}=\lambda_{s}^{*}$. In this way, we can obtain the admission fee $F$ as follows:

$$
F=\frac{\phi}{\lambda_{s}^{*}} R-\omega C_{s}
$$

Corresponding to the system parameters in Figs. $\mathbf{5}$ and $\mathbf{6}$, we provide numerical results of the admission fee $F$ in Table 9.

Table 9. Numerical results of the admission fee $F$

\begin{tabular}{|c|c|c|c|}
\hline $\begin{array}{c}\text { Arrival rate of PU } \\
\text { packets } \lambda_{p}\end{array}$ & $\begin{array}{c}\text { False alarm } \\
\text { probability } p_{f}\end{array}$ & $\begin{array}{c}\text { Mistake detection } \\
\text { probability } p_{m}\end{array}$ & $\begin{array}{c}\text { Admission fee } \\
F\end{array}$ \\
\hline \hline 0.12 & 0.02 & 0.05 & 3.985 \\
\hline 0.15 & 0.08 & 0.05 & 4.101 \\
\hline 0.15 & 0.08 & 0.05 & 4.226 \\
\hline 0.15 & 0.08 & 0.01 & 4.276 \\
\hline
\end{tabular}

\section{Conclusions}

In this paper, we addressed the question of ensuring various transmission quality of different kinds of user packets. This is achieved by proposing a novel spectrum allocation strategy with channel bonding mechanism for PU packets and channel reservation mechanism for SU packets. The appropriate system model is a three-dimensional Markov chain reflecting imperfect channel sensing results and the working principle of the proposed strategy. We gave a detailed analytical framework to derive estimation formulas for several performance measures. We also provided numerical results to validate the analysis and to investigate the number of the reserved (resp. licensed) channels on the system performance besides different arrival rate of PU packets, different arrival rate of SU packets, different mistake detection probability and different false alarm probability. Additionally, by analyzing the individually and socially optimal behaviors, we presented a charging policy for SU packets to optimize the system socially. 
In our future research, we will further study the joint optimation for the number of the reserved channels and the behaviors of SU packets.

\section{References}

[1] Hassan Al-Mahdi, Mohamed A. Kalil, Florian Liers, et al., "Increasing spectrum capacity for Ad-hoc networks using cognitive radios: An analytical model," IEEE Communications Letters, vol. 13, no. 9, pp. 676-678, September, 2009. Article (CrossRef Link)

[2] Beibei Wang and K. J. Ray Liu, “Advances in cognitive radio networks: A survey,” IEEE Journal of Selected Topics in Signal Processing, vol. 5, no. 1, pp. 5-23, February, 2011.

Article (CrossRef Link)

[3] Ying-Chang Liang, Kwang-Cheng Chen, Geoffrey Ye Li, et al., "Cognitive radio networking and communications: An overview," IEEE Transactions on Vehicular Technology, vol. 60, no. 7, pp. 3386-3407, September, 2011. Article (CrossRef Link)

[4] Pinki Yadav, Subhajit Chatterjee and Partha Pratim Bhattacharya, "A survey on dynamic spectrum access techniques in cognitive radio," International Journal of Next-Generation Networks, vol. 4, no. 4, pp. 27-46, December, 2012. Article (CrossRef Link)

[5] Minho Jo, Longzhe Han, Dohoon Kim, et al., "Selfish attacks and detection in cognitive radio Ad-hoc networks," IEEE network, vol. 27, no. 3, pp. 46-50, June, 2013. Article (CrossRef Link)

[6] Min Song, Chunsheng Xin, Yanxiao Zhao, et al., "Dynamic spectrum access: From cognitive radio to network radio," IEEE Wireless Communications, vol. 19, no. 1, pp. 23-29, February, 2012. Article (CrossRef Link)

[7] Saleem Aslam, Adnan Shahid, Kyung-Geun Lee, et al., "Primary user behavior aware spectrum allocation scheme for cognitive radio networks," Computers and Electrical Engineering, vol. 42, no. C, pp. 135-147, February, 2015. Article (CrossRef Link)

[8] Salameh, Haythem A. Bany, Krunz Marwan, et al., "Spectrum bonding and aggregation with guard-band awareness in cognitive radio networks," IEEE Transactions on Mobile Computing, vol. 13, no. 3, pp. 569-581, March, 2014. Article (CrossRef Link)

[9] Yafeng Wang, Chao Li, Tianwei Wen, et al., "Dynamic channel reservation for cognitive radio network," in Proc. of 2015 IEEE Int. Conf. on Computational Intelligence and Communication Technology, pp. 339-343, April, 2015. Article (CrossRef Link)

[10] Lei Jiao, Vicent Pla, Frank Y. Li, et al., "Analysis on channel bonding/aggregation for multi-channel cognitive radio networks," in Proc. of 2010 European Wireless Conf., pp. 468-474, 2010. Article (CrossRef Link)

[11] Shaunak Joshi, Przemyslaw Pawelczak, Danijela Cabric, et al., "When channel bonding is beneficial for opportunistic spectrum access networks," IEEE Transactions on Wireless Communications, vol. 11, no. 11, pp. 3942-3956, November, 2012. Article (CrossRef Link)

[12] Yasuharu Konishi, Hiroyuki Masuyama, Shoji Kasahara, et al., "Performance analysis of dynamic spectrum handoff scheme with variable bandwidth demand of secondary users for cognitive radio networks,” Wireless Networks, vol. 19, no. 5, pp. 607-617, July, 2013. Article (CrossRef Link)

[13] Indika A. M. Balapuwaduge, Lei Jiao, Vicent Pla, et al., "Channel assembling with priority-based queues in cognitive radio networks: Strategies and performance evaluation," IEEE transactions on wireless communications, vol. 13, no. 2, pp. 630-645, February, 2014. Article (CrossRef Link)

[14] Tamal Chakraborty and Iti Saha Misra, "An analytical framework for channel reservation scheme in cognitive radio network," in Proc. of the 2013 Int. Conf. on Advances in Computing, Communications and Informatics, pp. 127-132, 2013. Article (CrossRef Link)

[15] S. Lirio Castellanos-Lopez, Felipe A. Cruz-Perez and Genaro Hernandez-Valdez, "Channel reservation in cognitive radio networks with the restart retransmission strategy," in Proc. of the 7th Int. ICST Conf. on Cognitive Radio Oriented Wireless Networks and Communications, pp. 65-70, 2012. Article (CrossRef Link)

[16] Jian Wang, Aiping Huang, Wei Wang, et al., "Admission control in cognitive radio networks with finite queue and user impatience," IEEE Wireless Communications Letters, vol. 2, no. 2, pp. 175-178, April, 2013. Article (CrossRef Link) 
[17] Attahiru Sule Alfa, Queueing Theory for Telecommunications, New York: Springer, pp. 53-59, 2010. Article (CrossRef Link)

[18] Naishuo Tian and Zhe George Zhang, Vacation Queueing Models: Theory and Applications, Springer, 2006. http://dl.acm.org/citation.cfm?id=1211259

[19] Refael Hassin and Moshe Haviv, To Queue or not to Queue: Equilibrium Behaviour in Queueing Systems, Kluwer Academic Publishers, 2003. Article (CrossRef Link)

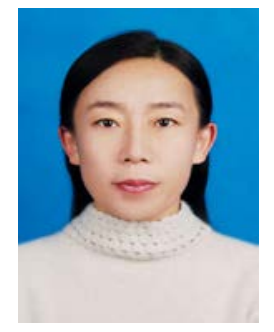

Shunfu Jin received the B.Eng. degree in computer and application from North East Heavy Machinery College, Qiqihaer, China, the M.Eng. degree in computer science and Dr.Eng. degree in circuit and system from Yanshan University, Qinhuangdao, China. Now Dr. Jin is a professor at School of Information Science and Engineering, Yanshan University, Qinhuangdao, China. Her research interests include stochastic modeling for telecommunication, performance evaluation for computer system and network, application for queueing system.

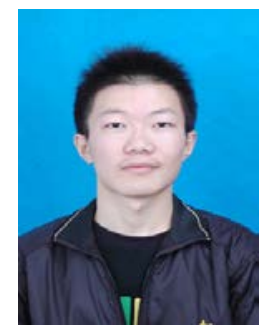

Xinghua Yao received the B.Eng. degree from Hebei Finance University, Baoding, Hebei, China. Now Mr. Yao is a postgraduate student at School of Information Science and Engineering, Yanshan University, Qinhuangdao, China. His reserch area is spectrum management in congitive radio networks.

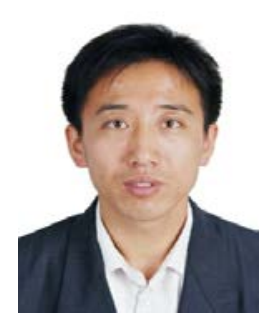

Zhanyou Ma received the B. Sc. degree in Mathematics from Jilin Normal University, Siping, China, the M. Sc. degree in operations research and Ph. D. in Management Science and Engineering from Yanshan University, Qinhuangdao, China. Now Dr. Ma is a professor at College of Sciences, Yanshan University, Qinhuangdao, China. His research interests include queueing systems with vacations and performance evaluation models in communication networks. 